\title{
Sepsis, Nicotine and the Cholinergic Anti-Inflammatory Pathway: A Literature Review
}

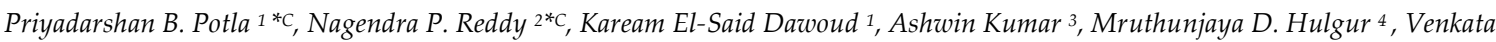

Budharaju ${ }^{5}$, Prathyusha Savarapu ${ }^{6}$, Avinash Mahindrakar ${ }^{7}$ and 8 Satyanarayana Oruganti.

Affiliations:

${ }^{1}$ Tameside E Glossop NHS Trust, Fountain St, Ashton-under-Lyne OL6 9RW, UK

${ }^{2}$ Care Medical LLC, 894 E 3900 S Ste B, Salt Lake City, UT 84107, USA

${ }^{3}$ Kempegowda Institute of Medical Sciences, Bengaluru, Karnataka 560070, India

${ }^{4}$ Royal Albert Edward Infirmary, Wigan Lane, Wigan, WN1 2NN, UK

${ }^{5}$ Florida Endocrinology E Diabetes Centre, 6229, 66 th Street, Florida 33781, USA

${ }^{6}$ Henry Ford Hospital, 2799, Detroit, Michigan 48202, USA

${ }^{7}$ Srikiran Institute of Opthammology, Kakinada, India

${ }^{8}$ Akshara Critical care group, Hyderabad, Telangana, 500072, India

${ }^{c}$ Corresponding and * First authors

Priyadarshan B. Potla

Tameside E Glossop NHS Trust

Ashton-under-Lyne. OL6 9RW

UK.

Darsh1911@gmail.com

Nagendra P. Reddy

Care Medical LLC, 894 E 3900 S Ste B

Salt Lake City, UT 84107

USA.

Prasad.reddy@carephysician.com

\section{Abstract}

The COVID-19 disease continues to disrupt modern life, escalating case fatality rates globally, posing arguably the greatest clinical challenge of the 21st century. Multiple pathophysiological mechanisms have thus far been suggested in the description of how SARS-CoV-2 may overcome the host's immune defence systems, and trials remain ongoing in the search for a cure. This article highlights the significant underrepresentation of smokers amongst COVID-19 in-patient cohorts, and emphasises the crucial role nicotine may serve through protective nicotinic anti-inflammatory pathways in attenuating the cytokine storm: the 'nicotinic hypothesis'. Previously poorly understood vagal-splenic mechanisms are further illustrated as this constitutes a key feature of the 'neuro-immune' axis, demonstrated to antagonise pro-inflammatory immune responses and may even reverse the signs and symptoms of sepsis. These unique findings synthesise the basis of the authors' recommendation for an urgent exploration of nicotine-based treatment modalities to combat this deadly pandemic.

Key words: COVID-19, SARS-CoV-2 Virus, Cholinergic Anti-inflammatory Pathway (CAP), Nicotine, $\alpha 7$ Nicotinic Acetylcholine Receptor ( $\alpha 7 \mathrm{nAchR})$, Vagus nerve. 


\section{Introduction}

The severe acute respiratory syndrome Coronavirus 2 (SARS-CoV-2), has spawned the grave COVID-19 pandemic, which has resulted in immense suffering and strain on global health resources on an unprecedented scale.

Frantic searches have been afoot on an unparalleled global level, rarely seen in modern times, in the hope of establishing the pathophysiology of this new disease to establish effective treatment modalities centred upon available contemporary knowledge, and the use of innovative strategies to contain or cure the disease. This article aims to focus the narrative on the role of the vagus nerve, the cholinergic anti-inflammatory pathway (CAP) and the $\alpha 7$ nicotinic acetylcholine receptor $(\alpha 7 \mathrm{nAChR})$, so-termed the 'neuro-immune axis'. And the influence of nicotine on this anti-inflammatory pathway, whereby raising our attention to the potential protective effects nicotine may exhibit in dampening down the well-recognised harmful hyper-stimulated cytokine-mediated immune response to reduce morbidity and mortality.

\section{The COVID-19 disease}

The clinical spectrum of presentation of COVID-19 varies from asymptomatic patients to severe respiratory failure, sepsis, septic shock, and multiple organ failure, with a case-fatality rate of $2.3 \%$ in confirmed cases [1]. The average time from the onset of symptoms to dyspnoea was noted to be five days; seven days to hospital admission and intensive care admission was on day eight [2]. Asymptomatic COVID-19 disease is observed in $40 \%$ to $45 \%$ of infected individuals, which suggests significant individual variability in the clinical picture of this disease [3]. Symptoms of SARS-CoV-2 infection included dry or productive cough, fever, lethargy, dyspnoea, headache, haemoptysis and diarrhoea [4]. Anosmia is a particular symptom and a diagnostic indicator in COVID-19 [5]. An increased risk of mortality was seen in elderly patients, diabetes, malignancies, hypertension, heart and chronic lung disease [6]. Patients with a poorer prognosis had very high levels of inflammatory cytokines, also known as the 'cytokine storm', and developed acute respiratory distress syndrome (ARDS) with impaired neuronal control of lung perfusion and hypoxic vasoconstriction [7].

\section{Smoking}

Smoking is a well-recognised detrimental factor in cardiovascular and respiratory diseases [8] via close effects on diverse nicotinic receptors present in the heart, blood vessels and lungs [9]. Smoking is also acknowledged in intensifying the morbidity and mortality secondary to common respiratory viral pathogens and the annual 
influenza virus [10]. However, the occurrence of some conditions like ulcerative colitis, sarcoidosis, farming and avian related inflammatory interstitial lung diseases, Parkinson's disease, Sjogren's syndrome [11] and preeclampsia [12] is surprisingly less common in smokers.

The considerable media attention thus far has attempted to highlight the assumptive negative association of smoking on COVID-19. However, current COVID-19 pandemic research has failed to demonstrate any significant relationship of the adverse influence of smoking on patients with COVID-19, such as increased hospitalisation or death [13].

Reflecting on China as a prominent case example, the origin of the outbreak [1], possessing a total population of 1.43 billion as of November 2019 [14] has a substantial smoking prevalence at $26.6 \%$ with roughly half of the entire male population as current smokers [15]. In striking contrast to widely held beliefs, active smokers contributed towards only a small fraction of confirmed COVID-19 cases in China [16]. In the United States, the country with the highest total numbers of COVID-19 cases as of June 2020 [17], data from May 2020 issued by the US Centre for Disease Control and Prevention (CDC) again demonstrated a considerable under-representation of smokers [18]; where the prevalence of smoking in the US is $13.8 \%$. In 7162 patients testing positive for the virus - only $1.3 \%$ were current smokers, $2.1 \%$ and $1.1 \%$ of hospitalised non-ICU patients and ICU admission were smokers, respectively [18].

Likewise, in 102 recent studies, there appears to be a surprisingly low prevalence of smokers among COVID-19 patients [13]. Simons et al. report that although current smokers displayed a lower incidence of a positive test, hospitalised smokers demonstrated an increased severity of COVID-19 disease manifestations requiring intensive care support and high mortality [13].

\section{Nicotinic Hypothesis}

The data across many countries so far reveal a significant inverse relationship between smoking and COVID-19 which is equally surprising and unexpected. However, the seminal and inspiring work performed by Tracey [19], Pavlov, Wang [20], Changeux [21], Borovikova [22] et al, may explain the perplexing underrepresentation of smokers among SARS-CoV-2 related hospital admissions and the unique benefit of nicotine in reducing the likelihood of severe infection with COVID19. Changeux et al. [23] coined the term: 'the nicotinic hypothesis', where the nicotinic acetylcholine receptor plays a vital function in the pathophysiology of COVID-19 disease, with the neurons of the olfactory system and the lungs as common sites for infection; thereby explaining the wide-ranging features of COVID19 disease [23]. The nicotinic hypothesis lends credence to nicotine as the protective agent in tobacco smoke [23]. Nicotine has been shown to markedly inhibit the production of pro-inflammatory cytokines like TNF- $\alpha$, interferon-gamma (IFN) and interleukin 2 (IL-2) following anti-CD3 stimulation, lending weight to the concept 
that nicotine is the principal immunomodulatory agent in tobacco [24]. Cytokines IL1, IL-6, IL-18 and TNF- $\alpha$ are secreted in large quantities following dysregulation of nicotinic Acetylcholine receptors $(\mathrm{nAChR})$ on bronchoalveolar macrophages, and the cytokine profile seen in the COVID-19 hyperinflammatory syndrome is remarkably similar to the $\mathrm{nAChR}$ dysregulation described [23]. The severe adverse outcome of smokers following hospital admission could be attributed to abrupt smoking cessation and the loss of nicotine's favourable influence [23]. Changeux suggests that the COVID-19 virus is a potent nicotinic agent and a nAChR blocker [23], and the nicotinic hypothesis directly challenges the current widely held school of thought purporting that the SARS-CoV-2 virus attaches to the cell wall angiotensinconverting enzyme 2 (ACE-2) receptor, to gain entry into the host cell, thereby triggering the dysregulation of the complex renin-angiotensin-aldosterone system [25].

The protective role of nicotine appears to manifest itself by direct action on the diverse groups of $\mathrm{nAChRs}$ present in the nervous system, immune cells, heart, lungs and blood vessels [23].

Interestingly, ivermectin, an anti-parasitic agent, recently considered for the treatment of COVID-19, has shown to prevent in-vitro COVID-19 replication [26] and is a positive allosteric modulator of $\alpha 7 \mathrm{nAChR}$ [27]. The anti-inflammatory effect of nicotine is also seen in Legionella pneumonia [28] and Chlamydia pneumonia [29].

Equally, nicotine has demonstrated to possess pharmacological properties via its anti-inflammatory action in diseases like Parkinson's disease, ulcerative colitis and Alzheimer's disease [30]. Nicotine has similarly prevented acute lung injury in animal ARDS models via its immunomodulatory effects [31].

\section{Acetylcholine}

Nicotine's naturally occurring biological equivalent is acetylcholine, an ester of acetic acid and choline, forming the dominant neurotransmitter of the cholinergic anti-inflammatory pathway [32]. Acetylcholine acts on the $\alpha 7$ subunit of nicotinic receptor $(\alpha 7 \mathrm{nAChR})$ which is a ligand-gated ion channel comprising of 5 subunits $\alpha 1-\alpha 10, \beta 1-\beta 4, \gamma, \delta$ and $\varepsilon 40$ on immune cells [33].

Acetylcholine then mediates complex intracellular signalling pathways following binding at the nicotinic (ligand-gated ion channels) or muscarinic receptor (Gprotein-coupled receptors) [33].

\section{The Neuro-Immune Axis}

The last few decades have afforded a more detailed insight into the interaction between the nervous system and inflammation: termed the 'neuro-immune axis'. This 'axis' is dependent upon the involvement of acetylcholine, the $\alpha 7$-nicotinic acetylcholine receptor ( $\alpha 7 \mathrm{nAChR})$ and the autonomic nervous system-controlled 
nicotinic anti-inflammatory pathway [19]. Tracey (2002) described the neuroimmune axis as a potent anti-inflammatory pathway, whereby the central nervous system through the vagus nerve regulates inflammation in the body following a sustained insult such as infection or trauma and can cause marked rapid inhibition of macrophage tumour necrosis factor (TNF- $\alpha$ ) release, with attenuation of the systemic inflammatory responses [19]. The nicotinic $\alpha 7$ acetylcholine receptors on immune cells serve as the primary mediators of this cholinergic anti-inflammatory pathway [19].

The archetypal diffusion-based anti-inflammatory group also called the "classical anti-inflammatory pathway", consists of insulin, glucocorticoids, oestrogens,

glucagon, thyroid hormones and other humoral agents [34].

An increased concentration of glucocorticoids seen in the early stage of inflammation, produces a down-regulation of inflammation [34], and oestrogens cause a marked reduction in inflammation following injury in several experimental models [34]. Furthermore, glucagon and thyroid hormones mediated by the adrenal cortex exert an indirect anti-inflammatory effect [34]. However, this classical antiinflammatory pathway is slow to act; concentration gradient dependant and largely non-integrated compared to the cholinergic anti-inflammatory pathway through its hard-wired lightning-fast neural connections [35].

\section{Inflammation}

Severe sepsis and multiorgan failure due to an exaggerated immune response is the principal cause of mortality in intensive care units in the industrialised world [36]. It has been noted in several cases that rather than the lethality of the primary offending organism, collateral damage following an exaggerated immune response is one cause of death in infectious disease [37]. Along with multiorgan failure, severe sepsis also causes dysregulation of coagulation, leading to disseminated intravascular coagulation [36,37].

The distinctive clinical signs of inflammation comprise of erythema (rubor), warmth (calor), swelling (tumor), and soreness (dolor) [38].

Inflammation serves as a crucial step in an efficient immune response with the repair of cellular injury and promotes pathogen identification; recruitment of immune cells; containment, pathogen clearance, and lastly, a return to baseline normal homeostasis [39].

Therefore, inflammation must be regulated at each stage to avert a hyperactive destructive systemic immune response, identified as the "cytokine storm", which is attributed to an overwhelming amount of pro-inflammatory cytokines, triggering the cardinal signs and symptoms of systemic inflammation, cardiovascular failure and life-threatening multiorgan failure [40]. Some pathogens, such as the influenza virus and Francisella tularensis, a Gram-negative bacterium, activate severe "cytokine storms" in patients, with significant major morbidity and fatality, where 
attenuating the inflammatory immune reaction in these conditions might be beneficial [41].

Counter-immune response mechanisms such as the cholinergic anti-inflammatory

pathway; cyclooxygenase pathways and platelet-activating inhibitory factor can limit the destructive effects of sepsis and cytokine storms, by reversal of positive inflammatory feedback circuits [41].

IL-10, TGF and several other cytokines have anti-inflammatory properties and serve to attenuate an inflammatory response [42]. Individual cytokines act on different phases of the inflammatory response, and for example, the T Helper-2 immune reaction cytokines diminish the T Helper-1 immune reaction and vice versa [43].

\section{Vagal Influence}

Our understanding of the intricate role through which the efferent vagal nerve diminishes the systemic inflammatory response and lethality continues to grow [44]. According to traditional doctrine, the sympathetic and the parasympathetic nervous systems work in opposition to each other [45]. However, in several conditions, the two systems work synergistically [45]. In a customary flight-or-fight response of the sympathetic nervous system, augmentation of vagal nerve output is commonly observed [45]. The combined effect of these two opposing autonomic nervous systems results in a significant anti-inflammatory effect, containment of local inflammation and prevention of the systemic spread of toxins [45].

Studies demonstrate vagus nerve stimulation downregulates pro-inflammatory immune responses and may even reverse the signs and symptoms of sepsis [46]. Following administration of lipopolysaccharide (LPS) an endotoxin, electrical vagus nerve stimulation significantly reduced harmful TNF- $\alpha$ levels in the serum and liver of rats [22]; thereby diminishing the secondary effects of severe sepsis and dysregulation of coagulation, resulting in increased survival rates [47]. Moreover, chemical or electrical vagus nerve stimulation in laboratory-induced endotoxaemia also revealed lower levels of TNF- $\alpha$ than expected, preventing septic shock; conversely, cervical vagotomy amplified serum TNF- $\alpha$ levels and accentuated the lethal noxious effects of LPS [48]. Interestingly, experimental vagus nerve stimulation in ischaemic reperfusion injury and haemorrhagic hypovolaemic shock prevented hypotension, similarly revealing low levels of TNF- $\alpha$ [22]. Additionally, vagal nerve stimulation reduced postoperative ileus and bowel inflammation secondary to surgical manipulation [49], and surgical vagotomy in rodents with septic and haemorrhagic shock increased the systemic inflammatory response [22]. Obesity and diabetes have been associated with severe COVID-19 disease, and studies have shown that in both conditions, the common denominator is reduced vagal activity. The reduced vagal activity, as seen in obesity and diabetes, could result in nAch receptor dysregulation resulting in the hyper-inflammatory state [50]. Vagus nerve stimulation is recognised to inhibit coagulation and fibrinolysis in the laboratory [47]. Therefore the procoagulant state seen in COVID-19 disease can be 
hypothesised as an imbalance between inflammation and the anti-inflammatory pathway.

\section{Splenic Involvement}

The spleen functions as an effector end-organ for the vagus nerve through facilitation of the systemic effects mediated through action on noradrenaline nerve endings in the spleen to inhibit the inflammatory response [51]. It has been shown that plasma T-lymphocytes act as a non-neural link between the vagus and the spleen, wherein the $\alpha 7 \mathrm{nACh}$ receptors in the spleen are vital for this inhibitory inflammatory response [51]. Moreover, post-splenectomy animals consistently failed to diminish serum TNF- $\alpha$ levels following vagal stimulation [52]. Nicotine has been shown to mimic vagus nerve stimulation and reduces mediators of inflammation in mice with an intact spleen; however, in splenectomised mice, nicotine caused a reverse effect with an increase in pro-inflammatory cytokines and lethality [52].

These observations confirm that the spleen forms an integral part of the cholinergic neuro-immune anti-inflammatory pathway [52].

\section{Conclusion}

In conclusion, despite the well-recognised harmful effects associated with smoking, smokers remain significantly under-represented in COVID-19 in-patient cohorts across the world and appear protected against the dangerous manifestations of the COVID-19 disease. Nicotine, a common substance found in tobacco, is a potent biological agonist at the $\alpha 7 \mathrm{nAChR}$, thus preventing entry of the SARS-CoV-2 virus into the host cell and a robust inhibitor of the inflammatory response. The authors have described the lightning-fast control of inflammation through hard-wired neural networks via the vagus nerve; the critical role of the $\alpha 7 \mathrm{nAChR}$ receptor as the principal fundamental receptor for the anti-inflammatory response and the significant anti-inflammatory effects of nicotine. The authors unequivocally stress that there remains no role for cigarette smoking as a treatment modality; however, nicotine as a chemical compound has validated laboratory evidence as a potent antiinflammatory agent. In these unprecedented times of COVID-19 related suffering and death, with no end in sight, the authors propose nicotine as an adjunct in the treatment modality for this deadly disease. The insight afforded in this article into the cholinergic anti-inflammatory pathway could reveal avenues to balance the inflammatory response; attenuate the hyper-inflammatory response of sepsis; avert the progression to life-threatening multiorgan failure, and save lives. 


\section{References}

1. Novel, C. P. E. R. E. (2020). The epidemiological characteristics of an outbreak of 2019 novel coronavirus diseases (COVID-19) in China. Zhonghua liu xing bing xue za zhi= Zhonghua liuxingbingxue zazhi, 41(2), 145.

2. D. Wang; B. Hu; C. Hu; F. Zhu; X. Liu; J. Zhang; B. Wang; H. Xiang; Z. Cheng; Y. Xiong; Y. Zhao; Y. Li; X. Wang; Z. Peng Clinical characteristics of 138 hospitalized patients with 2019 novel coronavirus-infected pneumonia in Wuhan, China, JAMA (2020)

3. Oran, D. P., \& Topol, E. J. (2020). Prevalence of Asymptomatic SARS-CoV-2 Infection: A Narrative Review. Annals of Internal Medicine.

4. Rothan, H. A., \& Byrareddy, S. N. (2020). The epidemiology and pathogenesis of coronavirus disease (COVID-19) outbreak. Journal of autoimmunity, 102433.

5. Heidari, F., Karimi, E., Firouzifar, M., Khamushian, P., Ansari, R., Ardehali, M. M., \& Heidari, F. (2020). Anosmia as a prominent symptom of COVID-19 infection. Rhinology, 58(3), 302303.

6. Wu Z, McGoogan JM. Characteristics of and important lessons from the coronavirus disease 2019 (COVID-19) outbreak in China: summary of a report of 72314 cases from the Chinese Center for Disease Control and Prevention. JAMA2020. doi:10.1001/jama.2020.2648 pmid:32091533

7. L. Gattinoni; S. Coppola; M. Cressoni; M. Busana; D. Chiumello Covid-19 does not lead to a 'typical' acute respiratory distress syndrome, Am. J. Respir. Crit. Care Med. (2020

8. Stratton, K., Shetty, P., Wallace, R., \& Bondurant, S. (2001). Clearing the smoke: the science base for tobacco harm reduction - executive summary. Tobacco control, 10(2), 189-195.

9. Kaur-Knudsen, D., Bojesen, S. E., Tybjærg-Hansen, A., \& Nordestgaard, B. G. (2011). Nicotinic acetylcholine receptor polymorphism, smoking behavior, and tobacco-related cancer and lung and cardiovascular diseases: a cohort study. Journal of clinical oncology, 29(21), 2875-2882.

10. Doolittle, L. M., \& Davis, I. C. (2018). Influenza in Smokers: More than Just a Cause of Symptom Exacerbations?.

11. Sopori, M. (2002). Effects of cigarette smoke on the immune system. Nature Reviews Immunology, 2(5), 372-377.

12. Chada, A. R., Lord, M., Nguyen, H. H. T., Davis, J. N., Bouzaher, M. H., Taylor, S. C., ... \& Chappell, J. C. (2020). 208: Impact of smoking on smooth muscle content of placental vessels in preeclampsia. American Journal of Obstetrics \& Gynecology, 222(1), S144.

13. Simons, D., Shahab, L., Brown, J., \& Perski, O. (2020). The association of smoking status with SARS-CoV-2 infection, hospitalisation and mortality from COVID-19: A living rapid evidence review. Qeios.

14. ^ Overall total population" - World Population Prospects: The 2019 Revision" (xslx). population.un.org (custom data acquired via website). United Nations Department of Economic and Social Affairs, Population Division. Retrieved 9 November 2019.

15. World Health Organization (WHO). Global Adult Tobacco Survey (GATS). Fact sheet China 2018.

16. Farsalinos, K., Barbouni, A. \& Niaura, R. Systematic review of the prevalence of current smoking among hospitalized COVID-19 patients in China: could nicotine be a therapeutic option? Intern Emerg Med (2020).

17. COVID-19 Worldwide Dashboard I WHO Live World Statistics

18. Centers for Disease Control and Prevention. Preliminary Estimates of the Prevalence of Selected Underlying Health Conditions Among Patients with Coronavirus Disease 2019 United States, February 12-March 28, 2020. MMWR Morb Mortal Wkly Rep 2020.

19. Tracey, K. J. (2002). The inflammatory reflex. Nature, 420(6917), 853-859. 
20. Pavlov, V. A., Wang, H., Czura, C. J., Friedman, S. G., \& Tracey, K. J. (2003). The cholinergic anti-inflammatory pathway: a missing link in neuroimmunomodulation. Molecular medicine, 9(5), 125-134.

21. Changeux, J. P., Devillers-Thiery, A., \& Chemouilli, P. (1984). Acetylcholine receptor: an allosteric protein. Science, 225(4668), 1335-1345.

22. Borovikova LV, Ivanova S, Zhang MH, Yang H, Botchkina GI, Watkins LR, Wang HC, Abumrad N, Eaton JW, Tracey KJ. 2000. Vagus nerve stimulation attenuates the systemic inflammatory response to endotoxin. Nature 405:458-462.

23. Changeux JP, Amoura Z, Rey F, Miyara M. A nicotinic hypothesis for COVID-19 with preventive and therapeutic implications [published online ahead of print April 22, 2020]. Qeios. 2020:FXGQSB.2. doi:10.32388/FXGQSB.2.

24. Ouyang, Y., N. Virasch, P. Hao, M. T. Aubrey, N. Mukerjee, B. E. Bierer, and B. M. Freed. 2000. Suppression of human IL-1_ IL-2, IFN-_ and TNF-_ production by cigarette smoke extracts. J. Allergy Clin. Immunol. 106:280.

25. Vaduganathan M, Vardeny O, Michel T, McMurray JJV, Pfeffer MA, Solomon SD. ReninAngiotensin-Aldosterone System Inhibitors in Patients with Covid-19. N Engl J Med. 2020. doi: 10.1056/NEJMsr2005760.

26. L. Caly; J. D. Druce; M. G. Catton; D. A. Jans; K. M. Wagstaff The FDA-approved Drug Ivermectin inhibits the replication of SARS-CoV-2 in vitro, Antiviral Res, Volume 2020, 104787

27. R. M. Krause; B. Buisson; S. Bertrand; P. J. Corringer; J. L. Galzi; J. P. Changeux; D. Bertrand Ivermectin: a positive allosteric effector of the alpha7 neuronal nicotinic acetylcholine receptor, MolPharmacol, Volume 53(1998), pp. 283-294

28. Matsunaga K, Klein TW, Friedman H, Yamamoto Y. 2001. Involvement of nicotinic acetylcholine receptors in suppression of antimicrobial activity and cytokine responses of alveolar macrophages to Legionella pneumophila infection by nicotine. J. Immunol. 167:65186524.

29. Mamata Y, Hakki A, Yamamoto Y, Newton C, Klein TW, Pross S, Friedman H. 2005. Nicotine modulates cytokine production by Chlamydia pneumoniae infected human peripheral blood cells. Int. Immunopharmacol. 5:749 -756.

30. Li, M. D. (2018). Nicotine modulates innate immune pathways via $\alpha 7$ nicotinic acetylcholine receptor. In Tobacco Smoking Addiction: Epidemiology, Genetics, Mechanisms, and Treatment (pp. 287-302). Springer, Singapore.

31. Mabley, J., Gordon, S., \& Pacher, P. (2011). Nicotine exerts an anti-inflammatory effect in a murine model of acute lung injury. Inflammation, 34(4), 231-237.

32. Aoki, K., Murayama, K., \& Nishiyama, H. (1995). Cation- $\pi$ interaction between the trimethylammonium moiety and the aromatic ring within lndole-3-acetic acid choline ester, a model compound for molecular recognition between acetylcholine and its esterase: an X-ray study. Journal of the Chemical Society, Chemical Communications, (21), 2221-2222.

33. Miyazawa A, Fujiyoshi Y, Unwin N (2003). Structure and gating mechanism of the acetylcholine receptor pore. Nature 423: 949-955.

34. Garcia-Leme, J., \& Farsky, S. P. (1993). Hormonal control of inflammatory responses. Mediators of inflammation, 2.

35. Farsalinos K, Niaura R, Le Houezec J, et al. Editorial: Nicotine and SARS-CoV-2:COVID-19 may be a disease of the nicotinic cholinergic system. T oxicol Reports 2020;

36. Ahrens, T., \& Tuggle, D. (2004). Surviving severe sepsis: early recognition and treatment. Critical care nurse, 24(5), S2-S13.

37. Angus, D. C., \& Van der Poll, T. (2013). Severe sepsis and septic shock. N Engl J Med, 369, 840-851. 
38. Tracy, R. P. (2006). The five cardinal signs of inflammation: calor, dolor, rubor, tumor... and penuria (apologies to Aulus Cornelius Celsus, De medicina, c. AD 25). The Journals of Gerontology Series A: Biological Sciences and Medical Sciences, 61(10), 1051-1052.

39. Kulkarni, O. P., Lichtnekert, J., Anders, H. J., \& Mulay, S. R. (2016). The immune system in tissue environments regaining homeostasis after injury: Is "inflammation" always inflammation?. Mediators of inflammation, 2016.

40. Hotchkiss and Karl, 2003 Hotchkiss RS, Karl IE (2003). The pathophysiology and treatment of sepsis. N Engl J Med 348: 138-150.

41. D'Elia, R. V., Harrison, K., Oyston, P. C., Lukaszewski, R. A., \& Clark, G. C. (2013). Targeting the "cytokine storm" for therapeutic benefit. Clin. Vaccine Immunol., 20(3), 319-327.

42. Opal SM, Depalo VA. 2000. Anti-inflammatory cytokines. Chest 117:1162-1172.

43. Kidd, P. (2003). Th1/Th2 balance: the hypothesis, its limitations, and implications for health and disease. Alternative medicine review, 8(3), 223-246.

44. Tracey, K. J. (2007). Physiology and immunology of the cholinergic antiinflammatory pathway. The Journal of clinical investigation, 117(2), 289-296.

45. Pongratz, G., \& Straub, R. H. (2014). The sympathetic nervous response in inflammation. Arthritis research \& therapy, 16(6), 504.

46. Song XM, Li JG, Wang YL, Hu ZF, Zhou Q, Du ZH, Jia BH. 2008. The protective effect of the cholinergic anti-inflammatory pathway against septic shock in rats. Shock 30:468-472.

47. Van Westerloo DJ, Giebelen IAJ, Meijers JCM, Daalhuisen J, De Vos AF, Levi M, van der Poll T. 2006. Vagus nerve stimulation inhibits activation of coagulation and fibrinolysis during endotoxemia in rats. J. Thromb. Haemost. 4:1997-2002.

48. Bencherif, M., Lippiello, P. M., Lucas, R., \& Marrero, M. B. (2011). Alpha7 nicotinic receptors as novel therapeutic targets for inflammation-based diseases. Cellular and molecular life sciences, 68(6), 931-949.

49. De Jonge WJ, van der Zanden EP, The FO, Bijlsma MF, van Westerloo DJ, Bennink RJ et al. (2005). Stimulation of the vagus nerve attenuates macrophage activation by activating the Jak2-STAT3 signaling pathway. Nat Immunol 6: 844-851.

50. Pavlov, V. A., \& Tracey, K. J. (2012). The vagus nerve and the inflammatory reflex-linking immunity and metabolism. Nature Reviews Endocrinology, 8(12), 743.

51. Martelli, D., McKinley, M. J., \& McAllen, R. M. (2014). The cholinergic anti-inflammatory pathway: a critical review. Autonomic Neuroscience, 182, 65-69.

52. Huston et al., 2006 Huston JM, Ochani M, Rosas-Ballina M, Liao H, Ochani K, Pavlov VA et al. (2006). Splenectomy inactivates the cholinergic anti-inflammatory pathway during lethal endotoxemia and polymicrobial sepsis. J Exp Med 203: 1623-1628 